\title{
Energy Efficient Resource Allocation for MIMO SWIPT Broadcast Channels
}

\author{
Jie Tang ${ }^{1}$, Daniel K. C. So ${ }^{2}$, Arman Shojaeifard ${ }^{3}$ and Kai-Kit Wong ${ }^{3}$ \\ ${ }^{1}$ South China University of Technology, Email: eejtang@scut.edu.cn \\ ${ }^{2}$ University of Manchester, Email: d.so@manchester.ac.uk \\ ${ }^{3}$ University College London, Email: \{a.shojaeifard, kai-kit.wong\}@ucl.ac.uk.
}

\begin{abstract}
In this paper, we address the energy efficiency (EE) optimization problem for SWIPT multiple-input multiple-output broadcast channel (MIMO-BC) with time-switching (TS) receiver design. Our aim is to maximize the EE of the system whilst satisfying certain constraints in terms of maximum transmit power and minimum harvested energy per user. The coupling of the optimization variables, namely, transmit covariance matrices and TS ratios, leads to a EE problem which is non-convex, and hence very difficult to solve directly. Hence, we transform the original maximization problem with multiple constraints into a min-max problem with a single constraint and multiple auxiliary variables. We propose a dual inner/outer layer resource allocation framework to tackle the problem. For the inner-layer, we invoke an extended SWIPT-based BC-multiple access channel (MAC) duality approach and provide an iterative resource allocation scheme under fixed auxiliary variables for solving the dual MAC problem. A sub-gradient searching scheme is then proposed for the outer-layer in order to obtain the optimal auxiliary variables. Numerical results confirm the effectiveness of the proposed algorithms and illustrate that significant performance gain in terms of EE can be achieved by adopting the proposed extended BC-MAC duality-based algorithm.
\end{abstract}

\section{INTRODUCTION}

Energy harvesting $(\mathrm{EH})$ is considered a prominent solution for prolonging the lifetime of power-constrained wireless devices [1]. In addition to well-recognized renewable energy sources such as biomass, wind, and solar, wireless power transfer (WPT) has emerged as a new enabler for EH. With WPT, the transmitter can transfer energy to the receivers via ambient radio frequency (RF) electromagnetic waves [2].

The integration of RF-based EH capability in communication systems opens up the possibility for simultaneous wireless information and power transfer (SWIPT). This topic has attracted great attention in both academia and industry recently [3]. Most research works on SWIPT systems aim to maximize the rate or the harvested energy, or otherwise achieve a certain rate-energy balance. Nevertheless, the standalone maximization of the system throughput would inherently constitute to the highest network power consumption. This trend goes against global commitments for tackling the socalled capacity crunch in a sustainable and economically viable manner. On the other hand, the sole goal of maximizing the harvested energy may degrade the delivered information, and in turn quality of service (QoS). An alternative strategy is therefore to consider energy-efficiency (EE), which is nowadays considered a fundamental performance metric in the design and deployment of wireless networks [4]. In addition to the many works on the EE optimization problem for conventional communication setups, the maximization of EE has been recently considered in the context of SWIPT systems [5]-[7].

In contrast to previous literature on EE for SWIPT, such as multi-carrier OFDMA systems [8], MISO systems based on a fixed precoder such as ZF [6] or MMSE [7], in this paper, we address the EE optimization problem for SWIPTbased MIMO-broadcast channel (BC) where TS technique is employed. By coupling the optimization variables in terms of transmit covariance matrices and TS ratios, the EE maximization problem under consideration becomes non-convex. Hence, it is very difficult to obtain the system EE solution using direct methods. Hence, to tackle this problem, we transform the original optimization problem with multiple constraints into a min-max problem with a single constraint and multiple auxiliary variables. In order to tackle the transformed problem, we propose a dual inner/outer layer resource allocation framework. By invoking the conventional BC-multiple access channel (MAC) duality principle [9], we formulate a dual SWIPT-based MIMO-MAC EE optimization problem with fixed auxiliary variables and accordingly provide an iterative resource allocation algorithm based on the Dinkelbach method [10]. A sub-gradient approach is then proposed in order to reach the optimal auxiliary variables in the outer-layer.

\section{System Model And Problem Formulation}

The system consists of a BS with $N_{T}$ transmit antennas and $K$ users $k \in\{1,2, \ldots, K\}$ each with $N_{R}$ receive antennas. We denote the channel matrix from the BS to the $k^{t h}$ user as $\mathbf{H}_{k} \in C^{N_{r} \times N_{t}}$. Channel state information (CSI) is assumed to be perfectly known at the corresponding transmitter and receivers. Different from the conventional MIMO downlink system, each transmission block in the SWIPT-based MIMOBC system is divided into two orthogonal time slots, one for ID and the other for EH, per illustrated in in Fig. 1. In particular, the TS-based receiver periodically switches its operations between ID and EH. Hence, the received signal from the BS to the $k^{\text {th }}$ user before TS is written as

$$
\mathbf{y}_{k}=\mathbf{H}_{k} \mathbf{x}+\mathbf{n}_{k},
$$

where $\mathbf{n}_{k} \in C^{N_{r} \times 1}$ is the independent zero mean Gaussian noise with each entry $\mathcal{C N}\left(0, \sigma^{2}\right), \mathbf{x}$ is the transmitted signal 


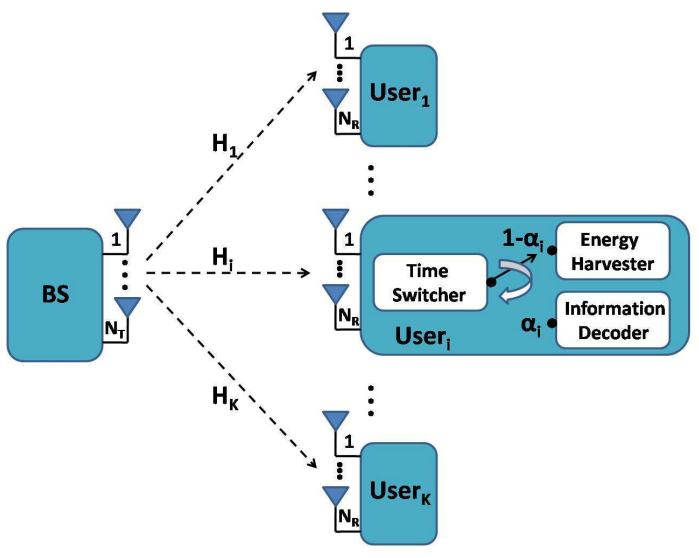

Fig. 1. A downlink SWIPT-based MIMO-BC system with TS-based receivers.

on the downlink. In addition, $\mathbf{x}=\mathbf{x}_{1}+\mathbf{x}_{2}+\ldots+\mathbf{x}_{K}$ where $\mathbf{x}_{k} \in C^{N_{t} \times 1}$, is the signal transmitted to the $k^{t h}$ user.

In this paper, receivers consisting of a harvesting energy unit and a conventional signal processing unit for concurrent $\mathrm{EH}$ and ID is under consideration. Let $\alpha_{k}$ with $0 \leq \alpha_{k} \leq 1$ denote the percentage of transmission time allocated to the ID time slot for user $k$. Thus, $1-\alpha_{k}$ corresponds to the percentage of transmission time allocated to the $\mathrm{EH}$ time slot for user $k$. Hence, the harvested energy at the receiver of user $k$ can be written as

$$
E_{k}=\left(1-\alpha_{k}\right) \eta_{k} \operatorname{tr}\left(\mathbf{G}_{k} \mathbf{Q}\right)
$$

where $\eta_{k}$ is a constant that accounts for the loss in the energy transducer for converting the harvested energy to electrical energy to be stored, $\mathbf{G}_{k}=\mathbf{H}_{k}^{H} \mathbf{H}_{k}$ is the channel covariance matrix, $\mathbf{Q}$ denotes the total transmit covariance matrix at the $\mathrm{BS}, \mathbf{Q}=\sum_{k=1}^{K} \mathbf{Q}_{k}^{b}, \mathbf{Q}_{k}^{b}=\mathbb{E}\left(\mathbf{x}_{k} \mathbf{x}_{k}^{H}\right)$ is the corresponding transmit covariance matrix, $\mathbf{Q}_{k}^{b} \succeq 0$ i.e., $\mathbf{Q}_{k}^{b}$ is a positive semidefinite matrix. On the other hand, the total capacity of the MIMO-BC SWIPT system can be expressed as follows

$$
C_{B C}=\sum_{k=1}^{K} \alpha_{k} R_{k}^{b}
$$

where $R_{k}^{b}$ denotes the rate achieved by the user $k$ in the downlink. Further, note that dirty paper coding (DPC) is the capacity achieving scheme for Gaussian MIMO-BC [11]. With DPC, the information for different users is encoded in a sequential fashion. It should be noted that the transmit covariance matrices remain the same during each transmission block. This implies that the same transmit covariance matrices are shared in ID and EH models. Without loss of generality, with an encoding order $(1, \cdots, K)$, i.e., the codeword of user 1 is encoded first, the data rate $R_{k}^{b}$ for the $k^{t h}$ user can be written as [9]

$$
R_{k}^{b}=W \log \frac{\left|\mathbf{I}_{N_{r} \times N_{r}}+\frac{1}{\sigma^{2}} \mathbf{H}_{k}\left(\sum_{i=k}^{K} \mathbf{Q}_{i}^{b}\right) \mathbf{H}_{k}^{H}\right|}{\left|\mathbf{I}_{N_{r} \times N_{r}}+\frac{1}{\sigma^{2}} \mathbf{H}_{k}\left(\sum_{i=k+1}^{K} \mathbf{Q}_{i}^{b}\right) \mathbf{H}_{k}^{H}\right|} .
$$

For the SWIPT-based MIMO-BC system considered in this work, the power consumption model should be extended considering EH devices. In general, small amounts of energy is consumed by the RF EH devices. On the other hand, the system power consumption is intuitively compensated by the harvested energy. It may then be apparent that enabling $\mathrm{EH}$ can improve the EE of a wireless communication system. Thus, as in [8], we take the harvested power into consideration in the formulation of the system power consumption model. Specifically, the total power consumption is given by

$$
P=\zeta P_{T}+P_{C}-\sum_{k=1}^{K} E_{k}
$$

where $\sum_{k=1}^{K} E_{k}$ represents the harvested power at all the receivers. Note that the minus sign denotes that a portion of the power radiated in the RF from the transmitter can be harvested by the $K$ receivers. Recall that $P_{C}$ is the total circuit power required for supporting reliable communication

$$
P_{C}=P_{a n t}^{B S} N_{T}+P_{s t a}+K P_{C}^{R},
$$

where $P_{a n t}^{B S} N_{T}$ denotes the power consumption proportional to the number of transmit antennas, $P_{s t a}$ is the constant signal processing circuit power consumption in the transmitters (due to filters, frequency synthesizer, etc., independent of the power radiated by the transmitter), and $P_{C}^{R}$ denotes the total circuit power consumption in the $K$ receivers.

The EE for SWIPT-based MIMO-BC can be defined as the total number of delivered bits per unit energy. Hence, we define EE in a SWIPT-based MIMO-BC as

$$
\lambda_{E E} \triangleq \frac{C_{B C}}{P}=\frac{\sum_{k=1}^{K} \alpha_{k} R_{k}^{b}}{\zeta P_{T}+P_{C}-\sum_{k=1}^{K} E_{k}},
$$

where $C_{B C}$ is the total capacity achieved by all users and $P_{T}=\sum_{k=1}^{K} \operatorname{tr}\left(\mathbf{Q}_{k}^{b}\right)$ is the total transmission power.

Given the expression of the system sum rate and power consumption, we can proceed with the problem formulation. The objective of this paper is to maximize the EE in SWIPTbased MIMO-BC whilst meeting two constraints in terms of transmission power and harvested energy. By invoking the linear power model in (5), the optimization problem can be formulated as

$$
\begin{aligned}
& \max _{\mathbf{Q}_{k}^{b} \succeq 0, \alpha_{k}} \frac{\sum_{k=1}^{K} \alpha_{k} R_{k}^{b}}{\zeta P_{T}+P_{C}-\sum_{k=1}^{K}\left(1-\alpha_{k}\right) \eta_{k} \operatorname{tr}\left(\mathbf{G}_{k} \mathbf{Q}\right)}, \\
& \text { s.t. } \quad\left(1-\alpha_{k}\right) \eta_{k} \operatorname{tr}\left(\mathbf{G}_{k} \mathbf{Q}\right) \geq E_{k, \min }, \forall k \in \mathcal{K}, \\
& \quad \sum_{k=1}^{K} \operatorname{tr}\left(\mathbf{Q}_{k}^{b}\right) \leq P_{\max }, \\
& \quad 0 \leq \alpha_{k} \leq 1, \forall k \in \mathcal{K}
\end{aligned}
$$

where $P_{\max }$ and $E_{k, \min }$ are the maximum total transmit power constraint at the BS and the minimum harvested energy constraint for user $k \in(1,2, \cdots, K)$, respectively. Note that (11) corresponds to the inherent constraints in terms of TS ratios. It is easy to see that the coupling of optimization variables leads to the problem (8)-(11) being non-convex and challenging to solve directly. Therefore, in the following sections, we develop resource allocation schemes for SWIPTbased MIMO-BC to solve the above optimization problem. 


\section{Equivalence AND Extended BC-MAC Duality}

In our SWIPT-based MIMO-BC setting, the EE optimization problem in (8)-(11) has not only a sum power constraint but also multiple minimum harvested energy constraints. The imposed multiple constraints complicates the formulation of an efficient solvable dual problem. In order to overcome the these challenges, we first transform this multi-constrained EE maximization problem into its equivalent problem that has a single constraint with multiple auxiliary variables. Thus, we develop a duality between a SWIPT-based MIMO-BC and a SWIPT-based dual MIMO-MAC in the case where the multiple auxiliary variables are fixed.

Proposition 1: Problem (8)-(11) shares the same optimal solution with the following equivalent problem

$$
\begin{aligned}
& \min _{\chi, \mu_{k}} \max _{\boldsymbol{Q}_{k}^{b}, \alpha_{k}} \frac{\sum_{k=1}^{K} \alpha_{k} R_{k}^{b}}{\zeta P_{T}+P_{C}-\sum_{k=1}^{K}\left(1-\alpha_{k}\right) \eta_{k} \operatorname{tr}\left(\boldsymbol{G}_{k} \boldsymbol{Q}\right)} \\
& \text { s.t. } \quad \chi\left(\sum_{k=1}^{K} \operatorname{tr}\left(\boldsymbol{Q}_{k}^{b}\right)-P_{\max }\right) \\
& +\sum_{k=1}^{K} \mu_{k}\left(E_{k, \text { min }}-\left(1-\alpha_{k}\right) \eta_{k} \operatorname{tr}\left(\boldsymbol{G}_{k} \boldsymbol{Q}\right)\right) \leq 0,
\end{aligned}
$$

where $\chi$ and $\mu_{k}$ are the auxiliary dual variables for the maximum power constraint and the $k^{\text {th }}$ minimum harvested energy constraint, respectively.

However, it is still very difficult to directly find an efficiently solvable dual problem for (12)-(13). Thus, in the following, we first investigate the EE maximization problem considering fixed auxiliary dual variables $\chi$ and $\mu_{k}$. The problem in (12)(13) can hence be reduced to

$$
\begin{aligned}
\max _{\mathbf{Q}_{k}^{b}, \alpha_{k}} & \frac{\sum_{k=1}^{K} \alpha_{k} R_{k}^{b}}{\zeta P_{T}+P_{C}-\sum_{k=1}^{K} \sum_{k=1}^{K}\left(1-\alpha_{k}\right) \eta_{k} \operatorname{tr}\left(\mathbf{G}_{k} \mathbf{Q}\right)} \\
\text { s.t. } & \chi\left(\sum_{k=1}^{K} \operatorname{tr}\left(\mathbf{Q}_{k}^{b}\right)-P_{\max }\right) \\
& +\sum_{k=1}^{K} \mu_{k}\left(E_{k, \text { min }}-\left(1-\alpha_{k}\right) \eta_{k} \operatorname{tr}\left(\mathbf{G}_{k} \mathbf{Q}\right)\right) \leq 0 .
\end{aligned}
$$

The solution of the above problem is unfortunately nontrivial given the objective function is non-concave even under fixed auxiliary dual variables $\chi$ and $\mu_{k}$. Thus, we exploit an extended SWIPT-based BC-MAC duality principle based on results from [9] and [12]. Consequently, the weighted sum rate maximization problem in SWIPT-based MIMO-BC under constraints in (13) can be formulated as

$$
\begin{aligned}
& \max _{\mathbf{Q}_{k}^{b} \succeq 0,0 \leq \alpha_{k} \leq 1} \sum_{k=1}^{K} \alpha_{k} R_{k}^{b}, \\
& \text { s.t. } \quad \chi \sum_{k=1}^{K} \operatorname{tr}\left(\mathbf{Q}_{k}^{b}\right)-\sum_{k=1}^{K} \mu_{k}\left(1-\alpha_{k}\right) \eta_{k} \operatorname{tr}\left(\mathbf{G}_{k} \mathbf{Q}\right) \leq P_{\text {all }},
\end{aligned}
$$

where $P_{\text {all }}:=\chi P_{\max }-\sum_{k=1}^{K} \mu_{k} E_{k, \min }$. Since $\chi$ and $\mu_{k}$ are fixed, $P_{\text {all }}$ is a constant (16)-(17). Hence, by extending the general BC-MAC duality principle from [9] to our SWIPTbased MIMO-BC scenario, we have the following SWIPT- based dual MAC problem corresponding to the original SWIPT-based BC problem in (16)-(17).

Proposition 2: The SWIPT-based dual MAC problem of (16)-(17) is given by

$$
\begin{aligned}
& \max _{\boldsymbol{Q}_{k}^{m} \succeq 0,0 \leq \alpha_{k} \leq 1} \sum_{k=1}^{K} \alpha_{k} R_{k}^{m} \\
& \text { s.t. } \quad \sum_{k=1}^{K} \operatorname{tr}\left(\boldsymbol{Q}_{k}^{m}\right) \leq P_{\text {all }},
\end{aligned}
$$

where $\boldsymbol{Q}_{k}^{m}$ is the transmit signal covariance matrix of the $k^{\text {th }}$ user, and $R_{k}^{m}$ is the rate achieved by the $k^{\text {th }}$ user of the dual MAC defined as

$$
R_{k}^{m}=W \log \frac{\left|\boldsymbol{N}+\boldsymbol{H}_{k}^{H}\left(\sum_{1}^{i} \boldsymbol{Q}_{i}^{m}\right) \boldsymbol{H}_{k}\right|}{\left|\boldsymbol{N}+\boldsymbol{H}_{k}^{H}\left(\sum_{1}^{i-1} \boldsymbol{Q}_{i}^{m}\right) \boldsymbol{H}_{k}\right|},
$$

with the noise covariance at the BS denoted with $\mathbf{N}=\chi \mathbf{I}-$ $\left.\sum_{k=1}^{K} \mu_{k}\left(1-\alpha_{k}\right) \eta_{k}\left[\operatorname{tr}\left(\boldsymbol{G}_{k} \boldsymbol{Q}\right)\right]\right)$.

Proposition 2 indicates that the capacity region of a SWIPTbased MIMO-BC with power constraint $P_{\text {all }}$ is equal to the union of capacity regions of the SWIPT-based dual-MAC with power constraints such that $\sum_{k=1}^{K} \operatorname{tr}\left(\mathbf{Q}_{k}^{m}\right)=P_{\text {all }}$. However, Proposition 2 describes the rate region for SWIPT-based MIMO-BC system and its duality relationship with SWIPTbased MIMO-MAC, meaning the EE aspect is still an open question. Hence, in order to tackle the problem in (14)-(15), we develop the following proposition (EE aspect) based on the results in Proposition 2.

Proposition 3: The solution of the SWIPT-based dual MAC EE maximization problem, namely,

$$
\begin{aligned}
& \max _{\boldsymbol{Q}_{k}^{m} \succeq 0,0 \leq \alpha_{k} \leq 1} \frac{\sum_{k=1}^{K} \alpha_{k} R_{k}^{m}}{\zeta \operatorname{tr}\left(\boldsymbol{Q}_{k}^{m}\right)+P_{C}} \\
& \text { s.t. } \quad \sum_{k=1}^{K} \operatorname{tr}\left(\boldsymbol{Q}_{k}^{m}\right) \leq P_{\text {all }},
\end{aligned}
$$

is an upper-bound of the solution to the problem in (14)-(15).

Consequently, instead of directly tackling the EE maximization problem in (14)-(15), in this work, we have provided a dual-MAC upper bound solution of (21)-(22).

To solve the optimization problem in (21)-(22), it is generally helpful to relate it to a concave program by separating numerator and denominator with the help of parameter $\beta$, this is what is known as the Dinkelbach method [10]. In the following sections, we propose an iterative resource allocation algorithm based on the Dinkelbach method to obtain the upperbound solution to the problem in (14)-(15).

\section{Iterative Resource Allocation Scheme based on DinKelbach Method}

Recall that the optimization problem in (21)-(22) belongs to a family of non-linear fractional programming problems which are non-convex and difficult to solve directly. Nevertheless, by invoking the theory of non-linear fractional programming in [10], we can use the Dinkelbach method to solve this nonconvex non-linear fractional programming problem. Specifically, based on Dinkelbach method [10], here, we propose an 


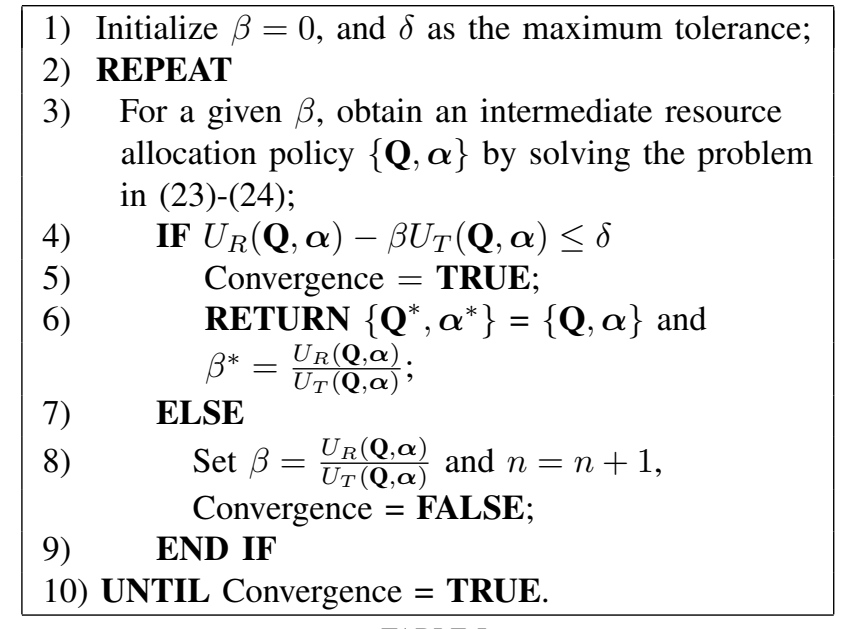

TABLE I

PROPOSED DINKELBACH METHOD-BASED SOLUTION

iterative algorithm for solving (21)-(22) with an equivalent objective function such that the obtained solution satisfies the conditions stated in Proposition 4. The proposed algorithm is summarized in Table I. It can be observed from Table I that the key step for the proposed iterative algorithm concerns the solution to the following optimization problem for a given parameter $\beta$ in each iteration (i.e., step 3),

$$
\begin{aligned}
& \max _{\mathbf{Q}_{k}^{m} \succeq 0,0 \leq \alpha_{k} \leq 1} \sum_{k=1}^{K} \alpha_{k} R_{k}^{m}-\beta\left(\zeta \sum_{k=1}^{K} \operatorname{tr}\left(\mathbf{Q}_{k}^{m}\right)+P_{C}\right) \\
& \text { s.t. } \sum_{k=1}^{K} \operatorname{tr}\left(\mathbf{Q}_{k}^{m}\right) \leq P_{\text {all }} .
\end{aligned}
$$

To solve this problem, we define $f\left(\mathbf{Q}_{1}^{m}, \cdots, \mathbf{Q}_{K}^{m}, \alpha_{1}, \cdots, \alpha_{K}\right) \quad=\quad \Delta_{k} \log \mid \mathbf{N}+$ $\sum_{k=1}^{K} \mathbf{H}_{k}^{H} \mathbf{Q}_{k}^{m} \mathbf{H}_{k} \mid$, where $\Delta_{k}=\alpha_{k}-\alpha_{k+1}$, and thus the optimization problem in (23)-(24) can be reformulated as

$$
\begin{array}{r}
\max _{\mathbf{Q}_{k}^{m} \succeq 0,0 \leq \alpha_{k} \leq 1} f\left(\mathbf{Q}_{1}^{m}, \cdots, \mathbf{Q}_{K}^{m}, \alpha_{1}, \cdots, \alpha_{K}\right) \\
-\beta\left(\zeta \sum_{k=1}^{K} \operatorname{tr}\left(\mathbf{Q}_{k}^{m}\right)+P_{C}\right) \quad \text { s.t. } \sum_{k=1}^{K} \operatorname{tr}\left(\mathbf{Q}_{k}^{m}\right) \leq P_{\text {all }} .
\end{array}
$$

The corresponding Lagrangian function can be expressed as

$$
\begin{aligned}
& L\left(\mathbf{Q}_{1}^{m}, \cdots, \mathbf{Q}_{K}^{m}, \alpha_{1}, \cdots, \alpha_{K}, \tau\right):=f\left(\mathbf{Q}_{k}^{m}, \alpha_{k}\right) \\
& -\beta\left(\zeta \sum_{k=1}^{K} \operatorname{tr}\left(\mathbf{Q}_{k}^{m}\right)+P_{C}\right)-\tau\left[\sum_{k=1}^{K} \operatorname{tr}\left(\mathbf{Q}_{k}^{m}\right)-P_{\text {all }}\right],
\end{aligned}
$$

where $\tau \geq 0$ is the Lagrangian multipliers associated with the maximum power constraint. The dual objective function of (23) is written as

$$
g(\tau)=\max _{\mathbf{Q}_{k}^{m} \succeq 0,0 \leq \alpha_{k} \leq 1} L\left(\mathbf{Q}_{1}^{m}, \cdots \mathbf{Q}_{K}^{m}, \alpha_{1}, \cdots, \alpha_{K}, \tau\right),
$$

and the dual problem is given by

$$
\min _{\tau} g(\tau) \quad \text { s.t. } \quad \tau \geq 0 .
$$

In this work, an iterative approach is used here in order to achieve the optimum $\mathbf{Q}_{k}^{m}$ and $\alpha_{k}$. In particular, we update
$\mathbf{Q}_{k}^{m}$ through the gradient of the Lagrangian function (26) with respect to $\mathbf{Q}_{k}^{m}$ and $\alpha_{k}$ as follows

$$
\begin{gathered}
\nabla_{\mathbf{Q}_{k}^{m}} L:=\frac{\partial f\left(\mathbf{Q}_{1}^{m}, \cdots, \mathbf{Q}_{K}^{m}\right)}{\partial \mathbf{Q}_{k}^{m}(n-1)}-\beta \zeta \tau \mathbf{I}_{N_{r} \times N_{r}}, \\
\nabla_{\alpha_{k}} L:=\frac{\partial f\left(\alpha_{1}, \cdots, \alpha_{K}\right)}{\partial \alpha_{k}(n-1)}, \\
\mathbf{Q}_{k}^{m}(n)=\left[\mathbf{Q}_{k}^{m}(n-1)+t \nabla_{\mathbf{Q}_{k}^{m}} L\right]^{+}, \\
\alpha_{k}(n)=\alpha_{k}(n-1)+t \nabla_{\alpha_{k}} L,
\end{gathered}
$$

where $t$ represents the step size, and the notation $[\mathbf{A}]^{+}$is defined as $[\mathbf{A}]^{+}:=\sum_{i}\left[q_{i}\right]^{+} \mathbf{v}_{i} \mathbf{v}_{i}^{H}$, with $q_{i}$ and $\mathbf{v}_{i}$ denote the $i^{\text {th }}$ eigenvalue and the corresponding eigenvector of $\mathbf{A}$ respectively. Therefore, we can compute the gradient in (29) and (30) as follows

$$
\begin{gathered}
\frac{\partial f\left(\mathbf{Q}^{m}\right)}{\partial \mathbf{Q}_{k}^{m}}=\mathbf{H}_{k}\left(\mathbf{I}_{N_{t} \times N_{t}}+\frac{1}{\sigma^{2}} \sum_{k=1}^{K} \mathbf{H}_{k}^{H} \mathbf{Q}^{m}{ }_{k} \mathbf{H}_{k}\right)^{-1} \mathbf{H}_{k}^{H}, \\
\left.\frac{\partial f(\boldsymbol{\alpha})}{\partial \alpha_{k}}=\sum_{i=1}^{K} \Delta_{i} \operatorname{tr}\left[\mu_{i} \eta_{i} \mathbf{G}_{i}\left(\sum_{k=1}^{i} \mathbf{N}+\mathbf{H}_{k}^{H} \mathbf{Q}_{k}^{m} \mathbf{H}_{k}\right)^{-1}\right)\right]+R_{k}^{m} .
\end{gathered}
$$

After we obtain the optimum $\mathbf{Q}_{k}^{m}$ and $\alpha_{k}$, our next task is to find out the optimal $\tau$. Given that the Lagrangian function $g(\tau)$ is a convex function with respect to $\tau$, we can achieve the optimal $\tau$ through a one-dimensional searching approach. Nevertheless, it is not guaranteed that $g(\tau)$ is differentiable, and thus the gradient approach may not available in this case. On the other hand, the sub-gradient approach can be applied to search the optimal solution where $\tau$ is updated in accordance with the sub-gradient direction as $P_{\text {all }}-\sum_{k=1}^{K} \operatorname{tr}\left(\mathbf{Q}_{k}^{m}\right)$.

Upon convergence of the transmit covariance matrices $\mathbf{Q}_{k}^{m}, k=1,2, \cdots, K$ and the TS ratios $\alpha_{k}, k=1,2, \cdots, K$, the current consumption power is saved in order to compare with $P_{\text {all }}$. In particular, the value of $\tau$ should be increased if $\sum_{k=1}^{K} \operatorname{tr}\left(\mathbf{Q}_{k}^{m}\right) \geq P_{\text {all }}$, and decrease otherwise. This procedure is continued until convergence, i.e., $\left|\tau_{\min }-\tau_{\max }\right| \leq \varepsilon$.

\section{Solution to the SWIPT-based EE Maximization PROBLEM}

Here, we provide a complete solution to the EE optimization problem in (12)-(13), namely an extended BC-MAC dualitybased EE maximization algorithm. Under fixed $\chi$ and $\boldsymbol{\mu}$, the problem can be reformulated as follows

$$
\begin{aligned}
& x(\chi, \boldsymbol{\mu})= \\
& \max _{\mathbf{Q}_{k}^{b}, \alpha_{k}} \frac{\sum_{k=1}^{K} \alpha_{k} R_{k}^{b}}{\zeta P_{T}^{m}+P_{C}-\sum_{k=1}^{K} \sum_{k=1}^{K}\left(1-\alpha_{k}\right) \eta_{k} \mathbb{E}\left[\operatorname{tr}\left(\mathbf{G}_{k} \mathbf{Q}\right)\right]} \\
& \text { s.t. } \chi\left(\sum_{k=1}^{K} \operatorname{tr}\left(\mathbf{Q}_{k}^{b}\right)-P_{\max }\right) \\
& +\sum_{k=1}^{K} \mu_{k}\left(E_{k, \text { min }}-\left(1-\alpha_{k}\right) \eta_{k} \mathbb{E}\left[\operatorname{tr}\left(\mathbf{G}_{k} \mathbf{Q}\right)\right]\right) \leq 0
\end{aligned}
$$

In addition, the problem (12)-(13) is equivalent as follows

$$
\min _{\chi, \boldsymbol{\mu}} x(\chi, \boldsymbol{\mu})
$$




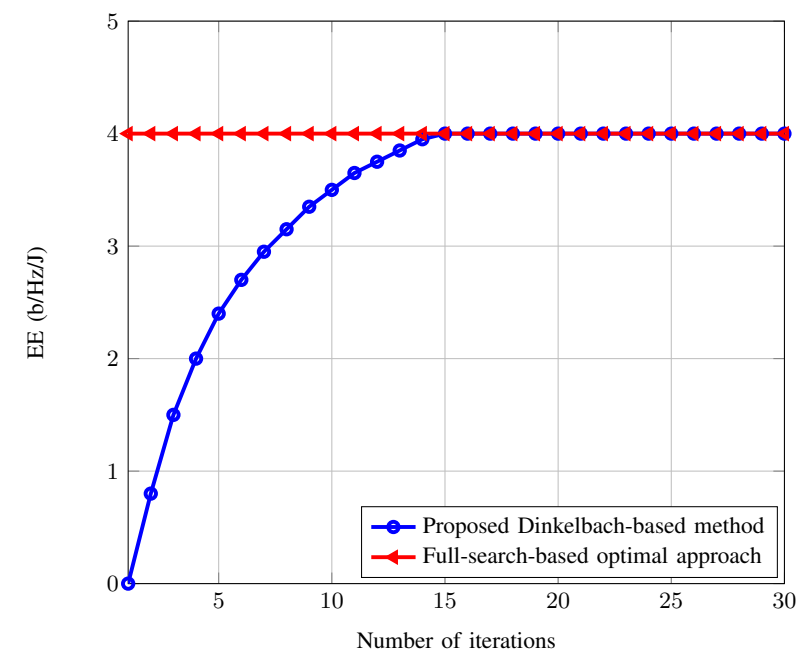

Fig. 2. Convergence behavior of the proposed Dinkelbach method-based scheme.

$$
\text { s.t. } \quad \chi \geq 0 \text { and } \mu_{k} \geq 0, \forall k \in \mathcal{K} \text {. }
$$

By applying the BC-MAC duality in Section III together with the proposed Dinkelbach method-based iterative resource allocation scheme in Section IV, one can achieve $x(\chi, \boldsymbol{\eta})$. We then apply the BC-MAC covariance mapping approach from [12] to obtain the corresponding BC transmit covariance matrices $\mathbf{Q}_{k}^{b}, k=1, \cdots, K$. Once we have obtained the solution for a given $\chi$ and $\boldsymbol{\mu}$, we can update $\chi$ and $\boldsymbol{\mu}$ through a sub-gradient approach. It should be noted that with a constant step size, the sub-gradient approach will converge to a point that is very close to the optimal value [13], i.e.,

$$
\lim _{n \rightarrow \infty}\left|\chi^{n}-\chi^{*}\right|<\epsilon, \text { and } \lim _{n \rightarrow \infty}\left|\mu_{k}^{n}-\mu_{k}^{*}\right|<\epsilon, k=1, \cdots, K,
$$

where $\chi^{*}$ and $\mu_{k}^{*}$ are the optimal values, and $\chi^{n}$ and $\mu_{k}^{n}$ are the values of $\chi$ and $\mu_{k}$ at the $n^{\text {th }}$ iteration of the subgradient approach, respectively. This result indicates that the sub-gradient approach determines an $\epsilon$-suboptimal point in a finite number of iterations.

\section{Simulation Results}

In our simulation, the BS employs $N_{t}=4$ transmit antennas, each user is equipped with $N_{R}=2$ receive antennas, and the total number of users is set to $K=4$. The path-loss is calculated using $128.1+37.6 \log _{10} d$ with distance $d$, and the radius of the cell is set to $500 \mathrm{~m}$. The drain efficiency of the power amplifier $\zeta$ is set to $38 \%$ in our simulation whilst the energy harvesting efficiency is set to $\eta=50 \%$. The power budget for each BS is considered to be $46 \mathrm{dBm}$ and the circuit power $P_{C}$ is $40 \mathrm{dBm}$. The minimum harvesting energy $E_{k}$ is set to $10 \%$ of the maximum transmit power.

In the first simulation, the performance of the proposed extended BC-MAC duality-based EE maximization algorithm is studied. The convergence behavior of the proposed Dinkelbach method-based scheme is first evaluated by illustrating how the EE performance behaves with the number of iterations. As shown in Fig. 2, the proposed Dinkelbach method-based

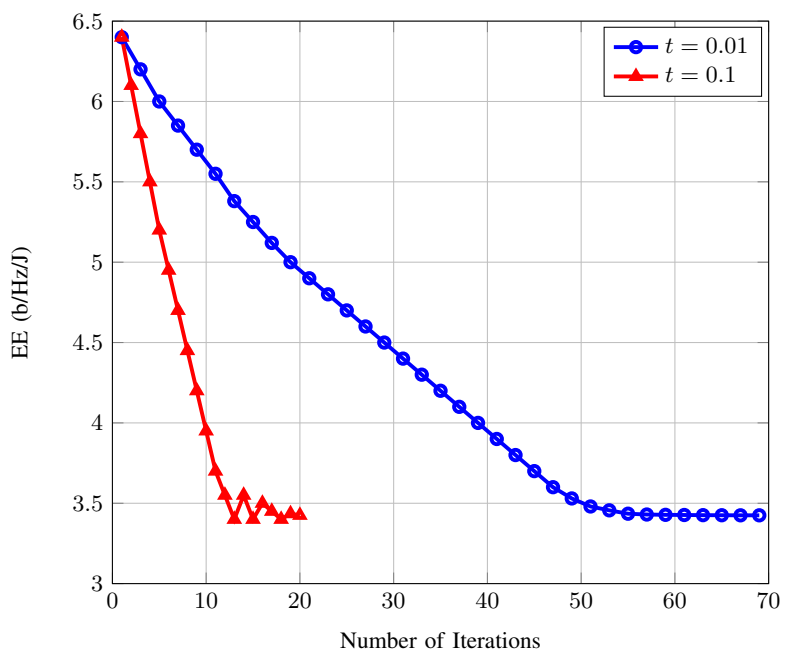

Fig. 3. Convergence behavior of the proposed extended BC-MAC dualitybased EE maximization algorithm in terms of EE.

iterative resource allocation scheme converges to the optimal value. In addition, the convergence behavior of the proposed upper bound sub-gradient resource allocation algorithm is also studied. Fig. 3 plots the EE versus the number of iterations for step sizes 0.1 and 0.01 . As can be seen from the figure, the proposed extended BC-MAC duality-based EE maximization algorithm converges to a stable value, and the step size affects the accuracy and convergence speed of the algorithm.

In the next simulation, the proposed extended BC-MAC duality-based EE maximization algorithm under different maximum transmit power allowance is evaluated and presented in Fig. 4. To show the EE gain achieved by TS-based SWIPT system, we compare our proposed scheme with the scheme that maximize the EE without $\mathrm{EH} \mathrm{[14]} \mathrm{and} \mathrm{the} \mathrm{scheme} \mathrm{that}$ aims for maximizing the system sum rate [15]. It is observed that the EE achieved by our proposed extended BC-MAC duality-based EE maximization algorithm is monotonically non-decreasing with respect to the maximum transmit power allowance $P_{\max }$. Particularly, the EE is increasing dramatically with an increasing maximum transmit power allowance $P_{\max }$ in the higher transmit power constraint region, i.e., $P_{\max }>25 \mathrm{dBm}$. This is because in the higher transmit power constraint region, a balance between the system EE and the total power consumption can be achieved. On the other hand, all the algorithms achieve similar performance in terms of the system EE criterion in the lower transmit power constraint region, i.e., $5<P_{\max }<15 \mathrm{dBm}$. Besides, due to the fact that the received power of the desired signal may not be sufficiently large for delivering information and energy harvesting at the same time, the system with TS-based energy harvesting receivers achieves a small performance gain compared to the system without energy harvesting receivers. Nevertheless, in the region of higher transmit power, the proposed extended BC-MAC duality-based EE maximization algorithm outperforms the other two schemes substantially. In particular, there is about a $5 \%$ gain can be achieved by our proposed extended BC-MAC duality-based EE maximization algorithm compared to the scheme that without energy harvesting receivers [14]. 


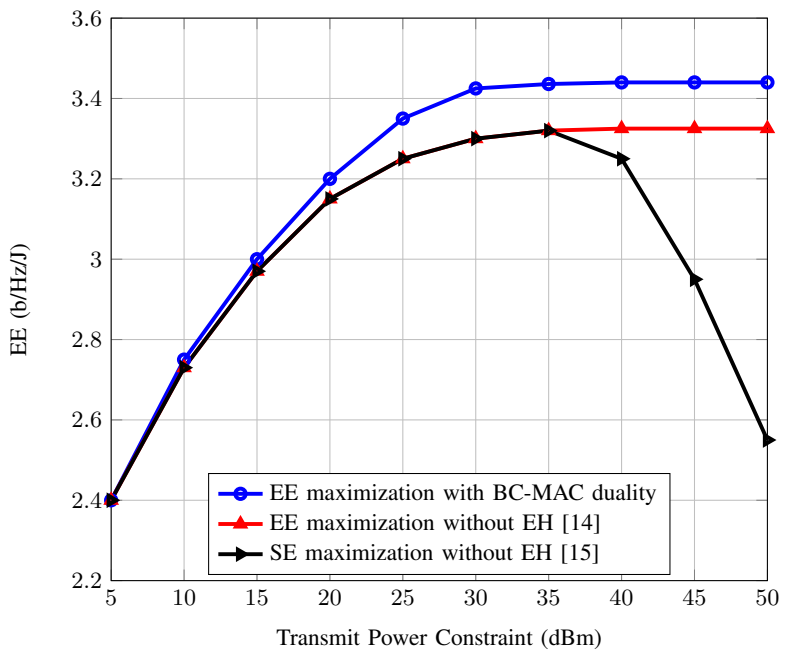

Fig. 4. The performance of the proposed extended BC-MAC duality-based EE maximization algorithm.

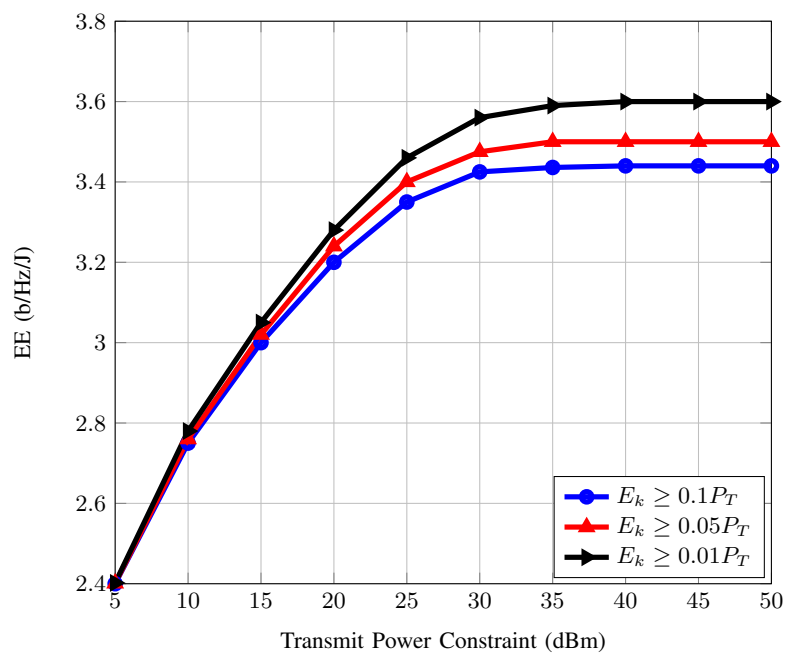

Fig. 5. Energy efficiency versus the maximum transmit power allowance for the proposed extended $\mathrm{BC}-\mathrm{MAC}$ duality-based $\mathrm{EE}$ maximization algorithm.

Furthermore, due to the fact that the increasing sum rate of the system cannot offset the consumption of the transmit power, the sum rate maximization scheme without energy harvesting [15] achieves a very low EE.

Finally, we investigate the system $\mathrm{EE}$ versus the maximum transmit power allowance for the proposed extended BC-MAC duality-based EE maximization algorithm with different level of minimum required harvested energy. As shown in Fig. 5, the increasing level of minimum required harvested energy will not always lead to an increasing system EE. Furthermore, jointly considering the results in Fig. 4 and Fig. 5, we can conclude that there exists an optimal minimum required harvested energy value for the EE optimization problem. Hence, the performance of EE can be further improved if the TS ratios and the minimum required harvested energy are jointly considered, and that would be investigated in our future works.

\section{CONCLUSIONS}

In this paper, we addressed the EE optimization problem for SWIPT-based MIMO-BC with TS receiver. The corresponding EE maximization problem from the coupling of the optimization variables, namely the transmit covariance matrices and TS ratios, is non-convex. Hence, to tackle the problem, we transform the original maximization problem with multiple constraints into a min-max problem with single constraint and multiple auxiliary variables. For the min-max problem with single constraint, a dual-layer resource allocation strategy is proposed. We incorporate an extended SWIPT-based BCMAC duality principle in order to simplify the inner-layer problem, and accordingly provide an iterative resource allocation algorithm for solving the dual MAC problem with fixed auxiliary variables. A sub-gradient-based searching scheme is then proposed to obtain the optimal auxiliary variables in the outer-layer. Numerical results validate the effectiveness of the proposed algorithms and show that significant performance gain in terms of EE can be achieved by our proposed extended BC-MAC duality-based EE maximization algorithm.

\section{REFERENCES}

[1] X. Lu, P. Wang, D. Niyato, D. I. Kim, and Z. Han, "Wireless networks with RF energy harvesting: A contemporary survey," Commun. Surveys Tuts., vol. 17, no. 2, pp. 757-789, 2nd Quart. 2015.

[2] I. Krikidis, S. Timotheou, S. Nikolaou, G. Zheng, D. W. K. Ng, and R. Schober, "Simultaneous wireless information and power transfer in modern communication systems," IEEE Commun. Mag., vol. 52, no. 11, pp. 104-110, Nov. 2014.

[3] Z. Xiang and M. Tao, "Robust beamforming for wireless information and power transmission," IEEE Wireless Commun. Lett., vol. 1, no. 2, pp. 372-375, Aug. 2012.

[4] Z. Hasan, H. Boostanimehr, and V. K. Bhargava, "Green cellular networks: A survey, some research issues and challenges," IEEE Commun. Surveys Tutorials, vol. 13, no. 4, pp. 524 - 540, Fourth Quarter 2011.

[5] D. W. K. Ng, E. S. Lo, and R. Schober, "Wireless information and power transfer: energy efficiency optimization in OFDMA systems," IEEE Trans. Wireless Commun., vol. 12, no. 12, pp. 6352-6370, Dec. 2013.

[6] Q. Shi, C. Peng, W. Xu, and M. Hong, "Energy efficiency optimization for MISO SWIPT systems with zero-forcing beamforming," IEEE Trans. Sig. Process., vol. 64, no. 4, pp. 842-854, Feb. 2016.

[7] S. He, Y. Huang, W. Chen, S. Jin, H. Wang, and L. Yang, "Energy efficient coordinated precoding design for a multicell system with RF energy harvesting," EURASIP J. Wireless Commun. Netw., vol. 67, 2015.

[8] D. W. K. Ng, E. Lo, and R. Schober, "Wireless information and power transfer: energy efficiency optimization in OFDMA systems," IEEE Trans. Wireless Commun., vol. 12, no. 12, pp. 6352-6370, Dec. 2013.

[9] S. Vishwanath, N. Jindal, and A. Goldsmith, "Duality, achievable rates, and sum-rate capacity of Gaussian MIMO broadcast channels," IEEE Trans. Inform. Theory, vol. 49, no. 10, pp. 2658 - 2668, Oct. 2003.

[10] W. Dinkelbach, "On nonlinear fractional programming," Management Science, vol. 13, pp. 492-498, Mar. 1967.

[11] H. Weingarten, Y. Steinberg, and S. S. (Shitz), "The capacity region of the Gaussian multiple-input multiple-output broadcast channel," IEEE Trans. Inform. Theory, vol. 52, no. 9, Sep. 2006.

[12] L. Zhang, Y. Xin, and Y. C. Liang, "Weighted sum rate optimization for cognitive radio MIMO broadcast channels," IEEE Trans. Wireless Commun., vol. 8, no. 9, pp. 2950 - 2959, June 2009.

[13] S. Boyd, L. Xiao, and A. Mutapcic, "Subgradient methods," Stanford University, 2003.

[14] J. Tang, D. K. C. So, K. A. H. E. Alsusa, and A. Shojaeifard, "On the energy efficiency-spectral efficiency trade-off in MIMO-OFDMA broadcast channels," IEEE Trans. Veh. Tech., vol. 65, no. 7, pp. 51855199, July 2016.

[15] J. Tang, K. Cumanan, and S. Lambotharan, "Sum-rate maximization technique for spectrum-sharing MIMO OFDM broadcast channels," IEEE Trans. Veh. Tech., vol. 60, no. 4, pp. 1960-1964, May 2011. 